\title{
Evolutionary State of "Field" Be Stars Deduced from BCD Parameters
}

\author{
J. Zorec ${ }^{1}$, Y. Frémat ${ }^{2}$, L. Cidale ${ }^{3}$, A.M. Hubert ${ }^{2}$, and M. Floquet ${ }^{2}$ \\ ${ }^{1}$ Institut d'Astrophysique de Paris, UMR7095/CNRS, 98 ${ }^{\text {bis }}$ bd. Arago, \\ F-75014 Paris, France (zorec@iap.fr) \\ ${ }^{2}$ Observatoire de Paris-Meudon, GEPI, FRE/2459, 92195 Meudon, \\ France \\ ${ }^{3}$ Facultad de Ciencias Astronómicas y Geofísicas, UN La Plata, \\ Argentina
}

\begin{abstract}
BCD $\left(\lambda_{1}, D\right)$ parameters of 49 "field" Be stars are used to show that the Be phenomenon may appear over the whole main sequence evolutionary span. Models of rotating $\mathrm{B}$ stars are used to estimate the incidence of the fast rotation in the $\left(\lambda_{1}, D\right)$ parameters.
\end{abstract}

\section{Aim and Observations}

The use of $\left(\lambda_{1}, D\right)$ parameters avoids to deal with perturbations introduced by the emissions/absorptions produced by the circumstellar envelope (CE) of $\mathrm{Be}$ stars on quantities employed to estimate their fundamental parameters and to discuss their evolutionary status. The evolutionary status of Be stars matters to understand whether their fast rotation is due to initial formation conditions, or it is an evolutionary consequence of their internal momentum redistribution mechanisms. The stellar sample used in the present contribution was presented in Zorec \& Briot (1991), which was frequently observed since 50 years, so that their $\left(\lambda_{1}, D\right)$ parameters are fairly well determined and reliable conclusions can be drawn from them. We assume, however, that each Be star studied evolves as a single object. Fig. 1a shows the HR diagram of the program Be stars given in terms of $\lambda_{1}$ (luminosity class or $\log g$-related quantity) and $D$ (spectral type

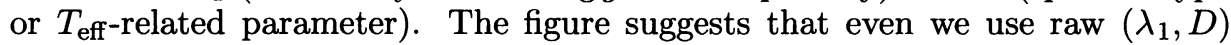
parameters, the Be phenomenon in "field" Be stars can appear at whatever evolutionary phase on the main sequence (MS), and, that the tendency for being in the second half of the MS is limited to late type Be stars.

\section{Models}

The rotationally induced stellar deformations were obtained for rigid rotation by solving Poisson's equation (Zorec et al. 1988) for the stellar internal density distributions with barotropic relations $P=P(\rho)(P=$ pressure; $\rho=$ density $)$ of non-rotating stars at different MS evolutionary phases for $\mathrm{Z}=0.02$ (Schaller et al. 1992). For the gravitational darkening effect we used von Zeipel's theorem. 

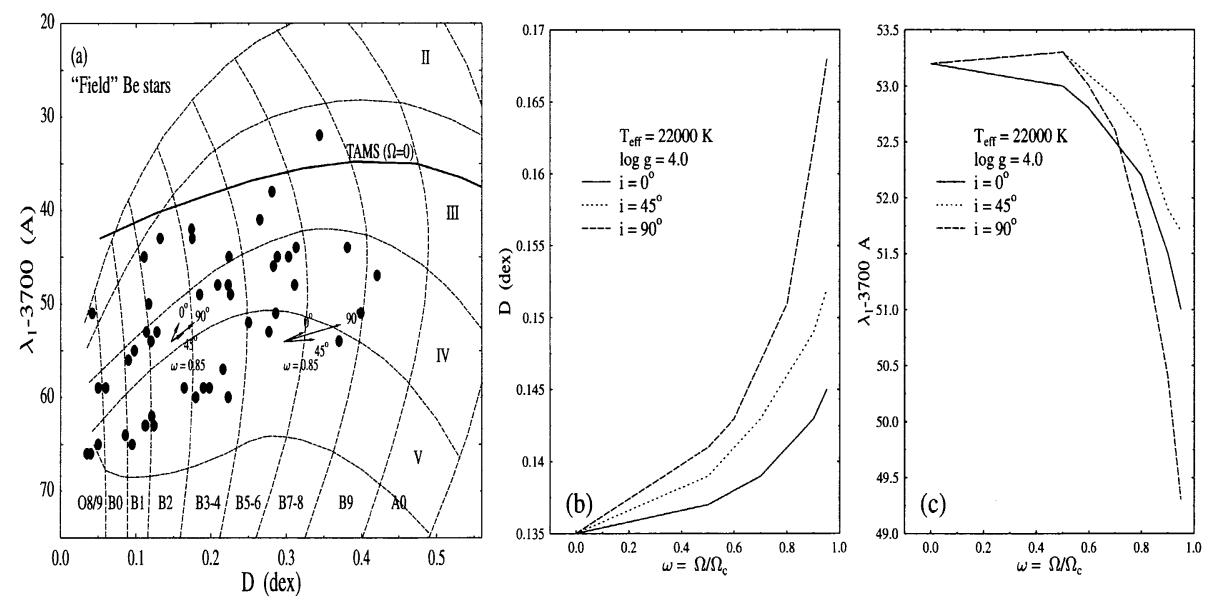

Figure 1. (a) HR diagram of Be stars in terms of observed $\left(\lambda_{1}, D\right)$ parameters. (b) and (c) respectively $D$ and $\lambda_{1}$ as a function of $\Omega / \Omega_{c}$ for different aspect angles $i, T_{\text {eff }}(\Omega=0)=22000 \mathrm{~K}$ and $\log g(\Omega=0)=4.0$

Figues $1 \mathrm{~b}$ and $1 \mathrm{c}$ show respectively the $\left(\lambda_{1}, D\right)$ parameters as a function of $\omega=\Omega / \Omega_{\mathrm{c}}$ (ratio of the angular velocity to the critical one) and $i$ (aspect angle) for initial unperturbed $T_{\text {eff }}=22000 \mathrm{~K}$ and $\log g=4.0$. In Fig. 1a are shown the displacements produced by $\omega=0.85$ at different $i$ in stars that would have rotationally unperturbed spectral types $\mathrm{B} 2 \mathrm{~V}$ and $\mathrm{B} 6 \mathrm{~V}$.

\section{Comments}

Rotationally induced instabilities and diffusioin of yields from the stellar core produce "blue-shifted" evolutionary tracks and enlarged stays of stars in the MS (Meynet \& Maeder 2000). It is expected then that the absolute age and mass estimates of our program stars may be somewhat underestimated. The stellar age ratios $t / t_{\text {TAMS }}$ for each star may however be still reliable, reflecting hence that the Be phenomenon in the earliest spectral types may appear in the whole $0<t / t_{\text {TAMS }}<1$ age ratio as suggested in Fig. 1a by the $\left(\lambda_{1}, D\right)$ parameters, though they are not corrected for rotational effects. The interval $0.5 \lesssim t_{\text {TAMS }} \lesssim 1$ seems to suite to late type Be stars.

\section{References}

Meynet G., Maeder A. 2000, A\&A 361, 101

Schaller G., Schaerer D., Meynet G., Maeder A. 1992, A\&AS 96, 269

Zorec J., Briot D. 1991, A\&A 245, 150

Zorec J., Mochkovitch R., Divan L. 1988, CRAS 306, 1265 